\title{
THE LINE SHAPE THEORY OF THE RADIOFREQUENCY SIZE EFFECT IN METALS*
}

\author{
E. A. KANER \\ Institute of Radiophysics and Electronics, Academy \\ of Science of Ukr.SSR, Kharkov, 85, U.S.S.R. \\ (Received 17 March 1967)
}

\begin{abstract}
The line shape theory of the radio frequency size effect for drift motion of an electron near a limiting point is developed. The investigation has been done taking account of diffuse electron scattering at the sample surface. There was established a good agreement of the theory and experiment. The coincidence of the calculated and measured line shape confirms the conclusions of anomalous skin-effect theory on the character of the field distribution in the skin-layer.
\end{abstract}

1. AT THE PRESENT the radiofrequency size effect (RSE) is widely used for the investigation of Fermi-surfaces and mean free paths of electrons in metals. The main point of these effects deals with the surface impedance of the plane parallel slab, which has structure of narrow line type in external magnetic fields.

All known RSE may be referred to two different groups. To the first one belongs the geometrical size effect caused by cutting the electron trajectories. The electron cut is observed any time when the extreme diameter trajectory $D_{\text {ext }}$ becomes equal to the slab thickness. This effect was for the first time predicted by the author when making theoretical investigations of the cyclotron resonance in plates [1]. Further it was discovered experimentally both at high [2], and low [3] frequencies. In a previous paper [4] there was developed the theory of line shape for RSE of geometrical type.

The second group of size effects is caused by trajectory type anomalous penetration (AP) of the electromagnetic field into metal $[5,6]$. The character of AP in this case is mainly different from the resonance excitation of collective, weak damping, electromagnetic waves [7]. The trajectory type AP field is connected with the motion of the separate electron groups. The effective electrons are those which move parallel to the metal surface in skin-layer. These electrons make narrow "splashes" (sheets) of alternating current and field which slowly damp into the metal. In the plane-parallel plate size effect lines arise when a "splash" appears on the second plate side.

The trajectory type AP field is caused by different mechanisms of discrimination of the

- The results of this paper were reported at the 10th International Congress on the Physics of Low Temperatures (Moscon, 1966). 
effective electrons. One of them is the mechanism of "the trajectory chain". The electron on the extreme cross-section of the Fermi surface does a finite motion in real space and makes a current sheet at a depth equal to the diameter $D_{0}$. For the electrons which are in the bulk of the metal, this splash serves as the original skin-layer which serves for the forming of the next splash at $2 D_{0}$, and so on. This AP field mechanism has been theoretically investigated [810]. "The trajectory chain" was for the first time discovered by Gantmakher in a size effect investigation in $\mathrm{Sn}[11]$.

The second mechanism of electron selection and splash formation is connected with the focusing of effective electrons during their drifting motion into a metal in an inclined magnetic field. The AP mechanism was first proposed by Gantmakher and Kaner [12]. In this paper it was shown that splashes are at the distances which are multiples of $u_{\text {ext }}$, where $u_{\text {ext }}$ is an extreme value of the normal projection of mean electron displacement in the period of motion in the magnetic field. The extremum of the function $u$ is necessarily achieved in the limiting point of the Fermi surface where the electron velocity is parallel to the magnetic field. The electrons in the vicinity of a limiting point move along a very drawnout helical trajectory (Fig. 1).

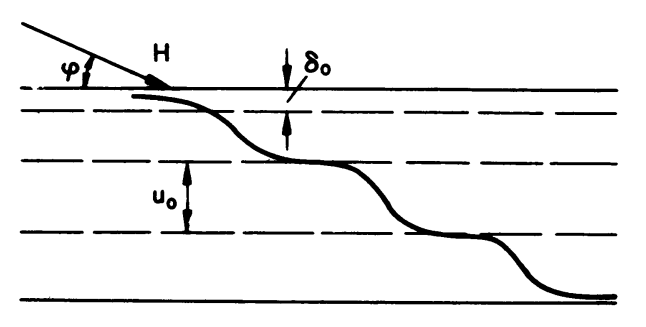

FIGURE 1

Electron trajectories in inclined magnetic field.

Splashes are where the electron velocity is parallel to a metal surface. In case of a complex Fermi surface other extrema of the mean displacement $u$ are possible. RSE from limiting points were found in $\mathrm{Sn}[12]$, In $[13 ; 14], \mathrm{Cd}[15], \mathrm{K}[16]$. The splashes arising in a metal from the limiting point cause interesting peculiarities concerning cyclotron resonance in inclined magnetic field $[10,17]$.

It is obvious that the RSE line shape directly reflects the character of the electromagnetic field distribution in the skin-layer. The investigation of this distribution is a very important problem of metal physics. Before the discovery of RSE direct methods of determination of the high frequency field damping and checking of the anomalous skin-effect theory did not exist.

The present paper is devoted to the theoretical investigation of RSE caused by the drifting motion of electrons in the vicinity of a limiting point. The comparison with the experimental results of Gantmakher and Krylov $[13,14]$ shows an excellent agreement of theory and experiment.

2. Usually in experiments an investigation of RSE in metals a sample is placed in a coil carrying an oscillating current and the real or imaginary part of the penetration depth $\delta_{H}$ of the alternating magnetic field $H_{\sim}$ v. external constant magnetic field $\mathbf{H}$ is measured. 


$$
\delta_{H}=\frac{1}{H_{\sim}(0)} \int_{0}^{d} d z H_{\sim}(z) .
$$

Here $d$ is a sample thickness, $\approx$ is the coordinate along the normal to surface. Very often not the value $\delta_{H}$ itself but its derivative $\partial \delta_{H} / \partial H$ is measured.

Under the condition of excitation from both sides there are skin-layers on both plate sides and the measured penetration depth $\delta_{H}$ is twice the thickness $\delta$ of each of the skin-layers.

$$
\delta_{H}=2 \delta, \quad \delta=\frac{1}{H_{\sim}^{(1)}(0)} \int_{0}^{d} d z H_{\sim}^{(1)}(z) .
$$

Here $H_{\sim}^{(1)}(z)$ is that part of the alternating magnetic field in the metal which is determined by slab excitation from one side. Using the Maxwell equation

$$
\operatorname{rot} \mathbf{E}=\frac{i \omega}{c} \mathbf{H}_{\sim}^{(1)} \text {, }
$$

the expression for $\delta_{H}$ may be given in the following form

$$
\delta_{H}=-2 \frac{E(0)-E(d)}{E^{\prime}(0)} .
$$

where $E(d)$ is the electric field in the second slab side connected with $\mathrm{AP}, E^{\prime}(0) \equiv d E(0) / d z$.

In the case of AP caused by limiting point electrons the amplitude of the transmitted wave is small in comparison with the field in the initial skin-layer. Therefore the effects of multiple reflection and interference of waves in the slab may be neglected. In these conditions the field distribution in the slab coincides with that in a semi-infinite metal. This distribution may be found from the Maxwell equation

$$
\frac{d^{2} E(t)}{d t^{2}}+\frac{4 \pi i \omega}{c^{2}} j(z)=0 .
$$

where $E(z)$ and $j(z)$ are the $y$-components of electric field and current density in the metal. Axis $y$ is parallel to the projection on the surface $z=0$ of the vector $H$. It is quite sufficient to consider only one equation (5) because the size effect from a limiting point takes place only for a small inclination of the vector $\mathbf{H}$ respective to the surface [12].

The current density is determined by the non-equilibrium part of electron distribution function $f$

$$
j=\frac{2 e}{(2 \pi \hbar)^{3}} \int v_{y} f d^{3} p .
$$

where $\mathbf{v}$ is the velocity, $\mathbf{p}$ is the momentum and $e$ is the absolute value of the electronic charge.

The function $f$ satisfies the Boltzmann equation, which we shall write in the relaxation time 
approximation:

$$
-i \omega f+v_{z} \frac{\partial f}{\partial z}+\Omega \frac{\partial f}{\partial \tau}+v f=e E(t) v_{y} \frac{\partial f_{0}}{\partial \xi}
$$

Here $f_{0}(\varepsilon)$ is the Fermi equilibrium distribution function, $\partial f_{0} / \partial_{\varepsilon}=-\delta\left(\varepsilon-\varepsilon_{F}\right), \varepsilon_{F}$ is the Fermi energy, $Q=e H / m c, m$ is the cyclotron mass, $m=\frac{1}{2 \pi} \cdot \frac{\partial S\left(\varepsilon F, p_{H}\right)}{\partial \varepsilon}, S$ is the cross-secti area of isoenergetic surface by the plane $p_{H} \equiv \mathbf{p H} / H=$ const., $T$ is the phase of the motion of the electron along its orbit in the magnetic field, and $v$ is the collision frequency. The possibility of introducing a relaxation time $1 / v$ under anomalous skin-effect conditions is discussed by Azbel" and Kaner [19].

It is necessary to add a boundary condition to equation (7). We shall consider the electron scattering to be diffuse,

$$
\left.f\right|_{v=0}=0
$$

According to [18], the solution of the equation (7) with condition (8) has the following form

$$
\begin{gathered}
f\left(\star, \tau, \varepsilon, p_{H}\right)=\frac{e}{\Omega\left(\varepsilon, p_{H}\right)} \frac{\partial f_{0}}{\partial \varepsilon} \int_{r\left(t, \tau, \varepsilon, p_{H}\right)}^{T} d \tau^{\prime} v_{y}\left(\tau^{\prime}\right) \exp \left[\gamma\left(\tau^{\prime}-\tau\right)\right] \cdot \\
\cdot E\left(\xi+\frac{1}{\Omega\left(\varepsilon, p_{H}\right)} \int_{\tau}^{T^{\prime}} d \tau^{\prime \prime} v_{*}\left(\tau^{\prime \prime}, \varepsilon, p_{H}\right)\right),
\end{gathered}
$$

where

$$
\gamma=v / \Omega<1
$$

We neglected the frequency $\omega$ of the external alternating field in comparison with $v$. The function $r\left(\xi, T, \varepsilon, p_{H}\right)$ means the moment of time $T$ when an electron collided with metal surface for the last time before it appeared at the point of phase space $z, T, \varepsilon, p_{H}$. This function is determined as the root of the equation

$$
\approx \Omega+\int_{T}^{r} d_{T}{ }^{\prime} v_{*}\left(T^{\prime}\right)=0 .
$$

which is the nearest to $T$. If this equation has no solution, then the function $r$ in the formulas (9) should be set equal $-\infty$.

When the magnetic field is directed at an angle to the metal surface it is impossible to find a simple expression for the function $r$ even in the simplest case of the Fermi-sphere. However it is clear from the further calculations that differences of the velocity projection $v_{z}$ from the mean value $\bar{v}_{z}$ are not very essential for the considered effect. So we shall substitute the mean value for $v_{z}$ in solving equation (11) and in boundary condition (8): 


$$
v_{*} \rightarrow \bar{v}_{*}=\frac{1}{2 \pi} \oint d \tau v_{*}(\tau)
$$

After this it is easy to find an approximate expression for the function

$$
r=\left\{\begin{array}{l}
-\infty,\left(\bar{v}_{*}<0\right) \\
\tau-\frac{\Omega_{*}}{v_{*}},\left(\bar{v}_{*}>0\right) .
\end{array}\right.
$$

If we substitute (9) in (6), we shall have

$$
\begin{gathered}
j(z)=\frac{2 e^{2}}{(2 \pi \hbar)^{3}} \int_{\varepsilon=\varepsilon_{F}} \frac{m d p_{H}}{\Omega} \oint d \tau v_{y}(\tau) \int_{r}^{T} d \tau^{\prime} v_{y}\left(\tau^{\prime}\right) \exp \left[\gamma\left(\tau^{\prime}-\tau\right)\right] \cdot \\
\cdot E\left(z+\Omega^{-1} \int_{\tau}^{\tau^{\prime}} d \tau^{\prime \prime} v_{*}\left(\tau^{\prime \prime}\right)\right) .
\end{gathered}
$$

We have changed integration over $d^{3} p$ to an integral over $d \tau d \varepsilon d p_{H}$. The Jacobian of this transformation is equal to $m$.

Now let us simplify expression (13) using the anomalous skin-effect conditions. As the skinlayer thickness is small compared to a typical value $v_{\tau} / \Omega$, the integrals over $T$ and $T^{\prime}$ may be calculated by the saddle point method. The saddle points are the roots of the equation

$$
v_{*}(\tau)=0 \text {. }
$$

At these points the electron velocities are parallel to metal surface for the effective electrons. Using the saddle point method in the integral over $T$ we shall get

$$
\begin{aligned}
\mid j(z)=\frac{2 e^{2}}{(2 \pi \hbar)^{3}} & \int \frac{m d p_{H}}{\Omega} \sum_{\tau_{\alpha}} v_{y}\left(\tau_{\alpha}\right) \int_{-\infty}^{\infty} d x \int_{\Gamma\left(z, \tau_{\alpha}\right)}^{\tau_{\alpha}} d \tau^{\prime} v_{y}\left(\tau^{\prime}\right) \\
\cdot & \exp \left[\gamma\left(\tau^{\prime}-\tau_{\alpha}\right)\right] E\left(z+\frac{1}{\Omega} \int_{\tau_{\alpha}}^{\tau^{\prime}} d_{\tau^{\prime \prime}} v_{z}\left(\tau^{\prime \prime}\right)-\frac{v_{z}^{\prime}\left(\tau_{\alpha}\right)}{2 \Omega} x^{2}\right) .
\end{aligned}
$$

Summation occurs over all $\tau_{\alpha}$ within the interval from 0 to $2 \pi$, In the integral over $\tau^{\prime}$ one saddle point coincides with the upper limit and all the others are within the interval of integration. In accordance with this we can write (15) in the following form

$$
j(z)=j_{0}(z)+\Delta j(z) \text {. }
$$

where $j_{0}(z)$ is a contribution from a saddle point $\tau^{\prime}=\tau_{\alpha}$ and $\Delta j(z)$ is the sum of all the other terms. First of all let us write the expression for $j_{0}(z)$ :

$$
j_{0}(z)=\frac{2 e^{2}}{(2 \pi \hbar)^{3}} \int \frac{m d p_{H}}{\Omega} \frac{1}{2} \sum_{\tau_{\alpha}} v_{y}{ }^{2}\left(\tau_{\alpha}\right) \int_{-\infty}^{\infty} \int_{-\infty} d x d y E\left(z+\frac{v^{\prime}\left(\tau_{\alpha}\right)}{2 Q}\left(y^{2}-x^{2}\right)\right) \text {. }
$$


The factor $1 / 2$ arose because of the fact that the integral over $y=\tau^{\prime}-\tau_{\alpha}$ is calculated from $-\infty$ to $+\infty$. It may be calculated from (17) that the double integral over $x$ and $y$ depends only on $\left|v_{*}^{\prime}\left(\tau_{\alpha}\right)\right|$. After evident changes of variables the formula (17) is represented in the following form

$$
j_{0}(z)=A_{y} \int_{-\infty}^{\infty} \int_{-\infty}^{\infty} d \xi d \eta E\left(z+\frac{1}{2}\left(\xi^{2}-\eta^{2}\right)\right) .
$$

where

$$
\begin{aligned}
& A_{y}=\frac{e^{2}}{(2 \pi \hbar)^{3}} \int d p_{H} \sum_{\tau_{\alpha}} v_{y}{ }^{2}\left(\tau_{\alpha}\right) /\left|v_{z}\left(\tau_{\alpha}\right)\right| \equiv \\
& \equiv \frac{e^{2}}{(2 \pi \hbar)^{3}} \int d^{3} p v_{y}^{2} \delta\left(v_{z}\right) \delta\left(\varepsilon-\varepsilon_{F}\right) .
\end{aligned}
$$

Integrating over the spherical image of the Fermi surface we get

$$
A_{y}=\frac{e^{2}}{(2 \pi \hbar)^{3}} \oint_{\vartheta=\pi / 2} \frac{d \lambda \cos ^{2} \lambda}{\mathrm{K}(\lambda)} \text {. }
$$

where $\lambda$ is an azimuthal angle in the velocity $\operatorname{space}\left(v_{z}=v(\vartheta, \lambda) \cos \vartheta, v_{y}=v(\vartheta, \lambda)\right.$ $\sin \vartheta \cos \lambda), \vartheta$ is the polar angle for which the poiar axis is parallel to $z_{\text {, }} K$-absolute value of the Gaussian curvature of Fermi surface.

All the rest of the saddle points $\tau^{\prime}=\tau_{\beta}<\tau_{\alpha}$ make a contribution to $\Delta j(z)$. For $\bar{v}_{z}<0$ the number of these points is infinite. Therefore the sum of such terms gives a small correction to $j_{0}(z)$ which smoothly depends on $z$. The singularity in current distribution arises from electrons with $\bar{v}_{z}>0$. When the value $z / u$ passes through a whole number, the number of saddle points changes by unity. The value

$$
u=2 \pi \bar{v}_{\boldsymbol{z}} / \Omega
$$

is a meen electron displacement for the period $2 \pi / \Omega$ along the axis $z$. Retaining only the one special term we can write

$$
\begin{gathered}
\Delta j(z)=\frac{2 e^{2}}{(2 \pi \hbar)^{3}} \int_{\bar{v}_{z}>0} \frac{m d p_{H}}{\Omega} \theta(z-n u) \sum_{\tau_{\alpha}} \frac{v_{y}^{2}\left(\tau_{\alpha}\right)}{\left|v_{z}^{\prime}\left(\tau_{\alpha}\right)\right|} \exp \left(-2 \pi \gamma \frac{z}{u}\right) \cdot \\
\cdot \int_{-\infty}^{\infty} d \xi d \eta E\left(z-n u+\frac{\xi^{2}-\eta^{2}}{2}\right),
\end{gathered}
$$

where $\theta(x)=1$ for $x>0$ and $\theta(x)=0$ for $x<0, n=[z / u]$ is the whole part of $z / u$.

Let us go from integrating over $p_{H}$ to integrating over $\lambda$ and take into consideration that the main role in the integral over $\lambda$ is played by a small vicinity of the limiting point $\lambda=0$, 
where the function $u$ has a maximum. Then

$$
\begin{gathered}
\Delta j(z)=\frac{2 e^{2}}{(2 \pi K)^{3}} \frac{\exp \left(-z / l_{0} \sin \varphi\right)}{K_{0}} \iint_{-\infty}^{\infty} \int_{-\infty} d \lambda d \xi d \eta \theta\left(z-n u_{0}-\frac{1}{2} n u_{0}{ }^{\prime \prime} \lambda^{2}\right) . \\
\cdot E\left(z-n u_{0}-\frac{1}{2} n u_{0}{ }^{\prime \prime} \lambda^{2}+\frac{\xi^{2}-\eta^{2}}{2}\right) .
\end{gathered}
$$

Here $l_{0}=2 \pi u_{0} / \gamma \sin \varphi$ is the mean free path at the limiting point.

The typical width of the integration region over $\lambda$ has the order $(\delta / z)^{1 / 2}<<1$. This small parameter determines also the respective size of $\Delta j(z)$ compared with $j_{0}(z)$. From formulas (23) it is clear that $\Delta j(z)$ is almost a periodic function $z$ with the period $u_{0}$, which exponentially damps at the distance $l_{0} \sin \varphi$.

Due to the fact that the correction $\Delta j(z)$ is small compared with $j_{0}(z)$ the equation (5) may be solved with the help of a perturbation method. For this aim let us formally continue the functions $E(z), j_{0}(z)$ and $\Delta j(z)$ evenly into the region $z<0$ and go over to Fourier-transforms. The equation (5) for the Fourier-components has the following form

$$
\kappa^{2} \mathrm{E}(\mathrm{K})+2 E^{\prime}(0)=\frac{4 \pi i \omega}{c^{2}}\left[\sigma_{0}(k) \mathrm{E}(\mathrm{k})+\Delta j(\kappa)\right] .
$$

where

$$
\begin{aligned}
E(\kappa)= & 2 \int_{0}^{\infty} d \kappa E(z) \cos \kappa z, \quad E(z)=\frac{1}{\pi} \int_{0}^{\infty} d \kappa E(\kappa) \cos \kappa z, \\
& \sigma_{0}(\kappa)=2 \pi A y /|\kappa| .
\end{aligned}
$$

Let us introduce the function

$$
T(z) \equiv-\frac{\pi}{2} \frac{E(z)}{E^{\prime}(0)} \equiv T_{0}(z)+\Delta T(z) .
$$

The zero' th approximation function

$$
T_{0}(z)=\int_{0}^{\infty} \frac{d \kappa \cos \kappa z}{\kappa^{2}-4 \pi i \omega c^{-2} \sigma_{0}(\kappa)} \equiv \delta_{0} \int_{0}^{\infty} \frac{d q q \cos \left(q z / \delta_{0}\right)}{q^{3}-i} .
$$

describes the field distribution in the skin-layer near the metal surface and coincides with the field distribution for $H=0$ [4]. The characteristic thickness of the skin-layer $\delta_{0}$ is determined by formulas

$$
5_{0}=\left(c^{2} / 8 \pi^{2} \omega A_{y}\right)^{1 / 3} .
$$

Figure 2 shows Re $T_{0}(z)$ and Im $T_{0}(z)$. It is clear that the zero' th approximation field differs essentially from zero at the distance $3-4 \delta_{0}$ from the surface. 

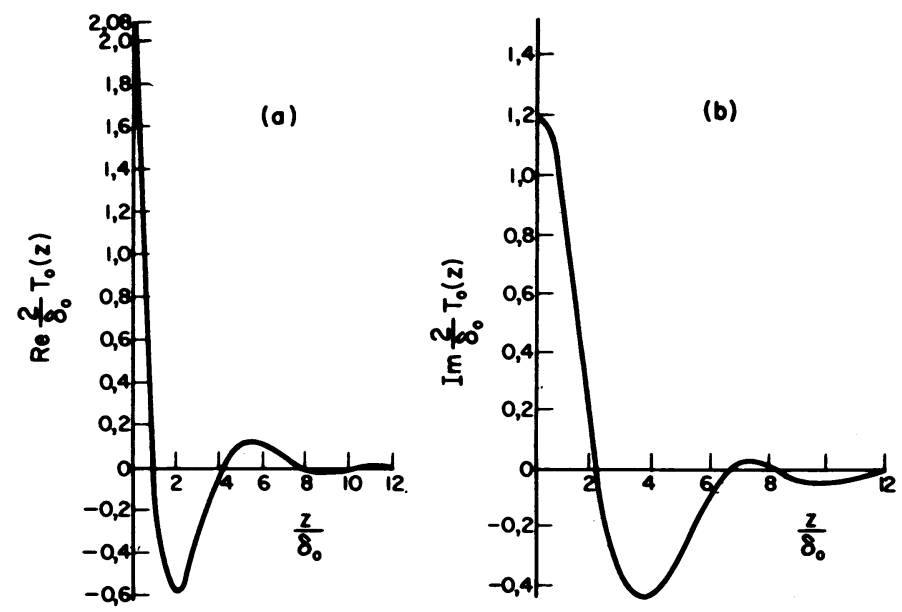

FIGURE 2

The dependence of real and imaginary parts of the function

$$
T_{0}(z) \text { from } t / \delta_{0}
$$

At large distances the electromagnetic field in the metal is described by the function

$$
\Delta T(*)=-\frac{2 \pi i \omega}{c^{2} E^{\prime}(0)} \int_{0}^{\infty} \frac{d k \cos k * \Delta j(k)}{k^{2}-4 \pi i \omega c^{-2} \sigma_{0}(k)}
$$

Express $\Delta j(k)$ with $\Delta j(z)$ using (25) and substitute in $\Delta j(z)$ the zero' th approximation for the field. Then

$$
\begin{aligned}
\Delta T(*)=\frac{4 i \omega}{c^{2}} & \int_{0}^{\varphi} d \zeta\left[T_{0}(z-\zeta)+T_{0}(z+\zeta)\right] \cdot \frac{2 e^{2} \exp \left(-\zeta / l_{0} \sin \varphi\right)}{(2 \pi \hbar)^{3} K_{0}} \\
\cdot \int_{-\infty}^{\infty} d \lambda \theta\left(\zeta-n u_{0}-\frac{1}{2} n u_{0}^{\prime \prime} \lambda^{2}\right) & \int_{-\infty}^{\infty} \int_{j}^{\infty} d \eta T_{0}\left(\zeta-n u_{0}-\right. \\
& \left.-\frac{1}{2} n u_{0}^{\prime \prime} \lambda^{2}+\frac{1}{2}\left(\xi^{2}-\eta^{2}\right) .\right) .
\end{aligned}
$$

We can neglect the term $T_{0}(*+\zeta)$ in these formulas and change the lower limit to - $\infty$ in the 
integral over $\zeta$. Besides, due to the sharp maximum of the function $T_{0}(z-\zeta)$ at $\zeta \simeq z$ the value $n=\left[\zeta / u_{0}\right]$ may be changed to $\left[t / u_{0}\right]$. After the substitution of the expression (27) for $T_{0}(t-\zeta)$ in (30) and calculating the integrals we get

$$
\Delta T(z)=-\left(\frac{8 \delta_{0}^{3}}{\pi}\right)^{x} \exp \left(-z / l_{0} \sin \varphi\right) \propto \Psi_{1}\left(\frac{z-n u_{0}}{\delta_{0}}\right) .
$$

where the nondimensional constant $\alpha$ is

$$
1 / \alpha=\left|\frac{u_{0}}{u_{0}{ }^{\prime \prime}}\right|^{1 / 2} \frac{\pi}{K_{0} \oint d \lambda \cos ^{2} \lambda K-1}
$$

For a Fermi-sphere $\alpha=1$.

The function $\Psi_{1}(x)$ is determined by the following formulae

$$
\begin{aligned}
\Psi_{1}(x)=\frac{1}{2 i} \int_{u}^{\infty} \frac{d q q^{1 / 3} \cos \left(q x+\frac{\pi}{4}\right)}{\left(q^{3}-i\right)^{2}}+\frac{1}{2 i} \int_{0}^{\infty} \frac{d q q^{1 / 3} \sin \left(q x+\frac{\pi}{4}\right)}{\left(q^{3}-i\right)\left(q^{6}+1\right)} . \\
\cdot\left[\frac{2}{\pi} q^{3} \ln q+\frac{2 q\left(q^{4}-1\right)}{\sqrt{ } 27}+\frac{i q}{3}\left(2 q^{2}+q+2\right)\right] .
\end{aligned}
$$

If we do not take into consideration the boundary condition (8) and use the expression for the distribution function in an infinite metal then $r=-\infty$ in formulas (9) for all the $v_{\text {. }}$. So in expression (23) for $\Delta j(z)$ the $\theta$-function is absent and the field distribution in the metal is described by formulas (31) in which $\Psi_{1}(x)$ is substituted for the function

$$
\Psi_{2}(x)=\frac{1}{i} \int_{0}^{\infty} \frac{d q q^{1 / 2} \cos \left(q x+\frac{\pi}{4}\right)}{\left(q^{3}-i\right)^{2}} .
$$

Figures 3 and 4 show the real and imaginary parts of the functions $\Psi_{1,2}\left[\left(z-n u_{0} / \delta_{0}\right)\right]$ and their derivatives with the boundary condition taken into account (curves 1 ) and without it (curves 2). These are obtained by numerical integration. From these curves it mas be concluded that the collisions of electrons with a metal surface essentially decrease the field at the left side of the splash (for $\approx<n u_{0}$ ) and practically do not change its appearance for $*>n u_{0}$. In other words the diffuse electron scattering cuts off the left side of the splash. This explains a known experimental fact $[13,16]$ that it is necessary to consider the left edge to be the real beginning of the line, but not the maximum position, when an impedance derivative is being measured. Besides it is obvious that the real and imaginary parts of the functions $\Psi_{1,2}(x)$

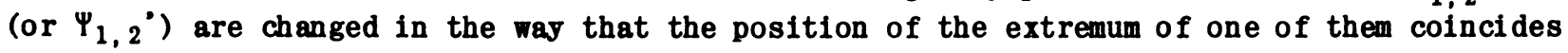
with the position of the most rapid change of the other.

The characteristic width in space of the field splashes in the bulk of a metal has an order of $6-8 \delta_{0}$ and is approximately twice the width of the function $T_{0}(z)$.

Let us disciss the application of the theory and the conditions for splashes occurring [12]. 
It is evident that the current sheets will be narrow only when the distances between the neighbouring lines are much greater than $\delta_{0}$. Thus

$$
\delta_{0} / p<\sin \varphi \text {. }
$$

Here $\rho$ is the displacement of the electron, that is near a limiting point for the period $2 \pi / \Omega$ along the vector $\mathbf{H}$. There exists a limitation on the side of large inclination angles, connected

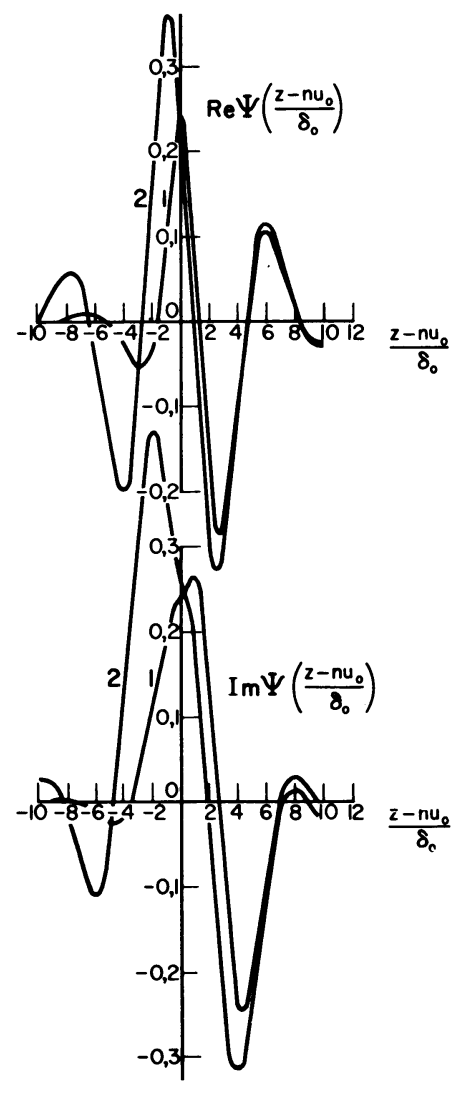

FIGURE 3

The dependence of real and imaginary parts of functions $\Psi_{1}$ (curves 1) and $\Psi_{0}$ (curves 2 ) on $\left(*-n u_{0}\right) / \delta_{0}$.

with the fact that at large angles electrons of a limiting point are not effective and the saddle point method cannot be applied for them. A sufficient criterion for existence of effective electrons at the limiting point has the form

$$
\Phi<\psi
$$

Here $\psi$ is characteristic angular dimension of the limiting point neighbourhoods that determine the changing of the drift velocity. The angle $\psi$ may be found from the condition that the space 
defocusing of electrons for a mean free time $1 / v$ has the order $\delta_{0}$, that is

$$
\frac{\Omega}{2 \pi v}\left|u(\psi)-u_{0}\right| \simeq \delta_{0}
$$

Consequently,

$$
\psi \sim\left(\delta_{0} / l_{0} \sin \phi\right)^{1 / 2}
$$

and the inequality (36) takes the following form*

$$
\sin \varphi<\left(\delta_{0} / l_{0}\right)^{1 / 3} .
$$

It is necessary to notice that the above developed theory of the AP field in metal is applicable not only to the case of a limiting point.

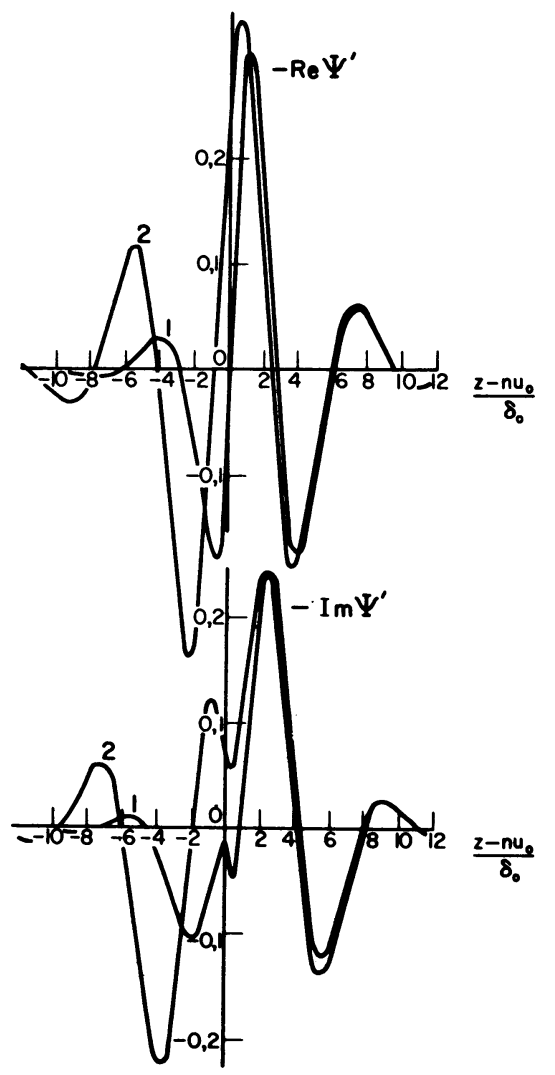

FIGURE 4

The dependence of real and imaginary parts of the derivatives $\Psi_{1}^{\prime}$ (curves 1 ) and $\Psi_{2}^{\prime}$ (curves 2) or $\left(t-n u_{0}\right) / \delta_{0}$.

* The more exact investigation that we shall not indulge in (details see in [6]) shows that the necessary condition is the unequality $\sin \varphi<\left(\delta_{0} p^{2} / l_{0}^{3}\right)^{1 / 7}$. Usually in an experiment both conditions practically coincide. 
The obtained formula correctly describe the field distribution in metals for focusing of other effective electron groups with the extreme displacement $u_{\text {ext }}$. Thus current sheets exist at any inclination angle $[12,15]$ of the magnetic field respective to a metal surface.

3. It is necessary to establish the connection between the field distribution in semi-infinite metals and the surface impedance of the plane-parallel slab for the comparison of the above developed theory with the experimental data. The surface impedance $Z=R-i X$ is connected with the penetration depth by the relation

$$
R-i X=-\frac{4 \pi i \omega}{c^{2}} 2 \delta .
$$

Taking the formulas (2), (4) and (26), we shall have

$$
R-i X=\frac{16 i \omega}{c^{2}}[T(d)-T(0)] \text {. }
$$

As was pointed out above impedance derivative is usually measured. From (31) we see

$$
\begin{aligned}
& \frac{d R}{d H}=\frac{16 \alpha \omega}{c^{2}}\left(\frac{8 \delta_{0} d}{\pi}\right)^{1 / 2} \frac{\exp \left(-d / l_{0} \sin \varphi\right)}{H} \operatorname{Im} \Psi_{1} \cdot\left(\frac{d-n u_{0}}{\delta_{0}}\right) . \\
& \frac{d X}{d H}=\frac{16 \alpha \omega}{c^{2}}\left(\frac{8 \delta_{0} d}{\pi}\right)^{1 / 2} \frac{\exp \left(-d / l_{0} \sin \varphi\right)}{H} \operatorname{Re} \Psi_{1}^{\prime}\left(\frac{d-n u_{0}}{\delta_{0}}\right) .
\end{aligned}
$$

From these formulas it is clear that the amplitude of the RSE lines for the derivative $d Z / d H$ decreases as $1 / H$. The position of the $n^{\prime}$ th line in the $H$-scale is proportional to the number

$$
H_{n}=n H_{1} \text {, }
$$

where $H_{1}$ is that magnetic field value for which $d=u_{0}$. Measuring line positions the value $u_{0}=\rho \sin \phi$ may be found. In this way the Gaussian curvature at the limiting point $\left(p=c K_{0}-1 / 2 / e H\right)$ mas be determined. The investigation of the angle dependence of line amplitude permits us to find the mean free path $l_{0}$ of electrons at a limiting point. And finally, from the comparison of the calculated and measured line shape the skin-depth $\delta_{0}$ may be found and the anomalous skin-effect theory checked.

Figure 5 shows the dependence of the derivative $\partial R / \partial H$. on $H$ which has been obtained by Gantmakher and Krylov [13] on an indium monocrystal. The experimental conditions are the following: $T=1.3^{\circ} \mathrm{K}$, inclination angle $\phi \sim 7^{\circ}$, magnetic field parallel to [111] axis, vector $\mathbf{E} 11$ [111], normal to the surface (axis $z$ ) parallel to [001], frequency $\omega / 2 \pi=3 \mathrm{Mc}$, slab thickness $0.3 \mathrm{~mm}$. From this figure is distinctly seen the periodicity of RSE lines. Their decay versus number is described by low $1 / n$ in accordance with formula (40).

Figure 6 shows the results of comparison of experimental and theoretical line shapes for $n=1$. As the scale of the experimental curves on the ordinate axis is arbitrary we tried to choose the scale on the theoretical curves to ensure the best coincidence on the right side of the lines. The pointed scale on the ordinate axis refers to - Im $\Psi_{1}^{\prime}\left(-\operatorname{Re} \Psi_{1}^{\prime}\right)$ respectively. It is clear that there is excellent agreement of both theoretical and experimental curves (the coincidence is somewhat worse for $\partial X / \partial H)$. The disagreement at the left side of the line is 


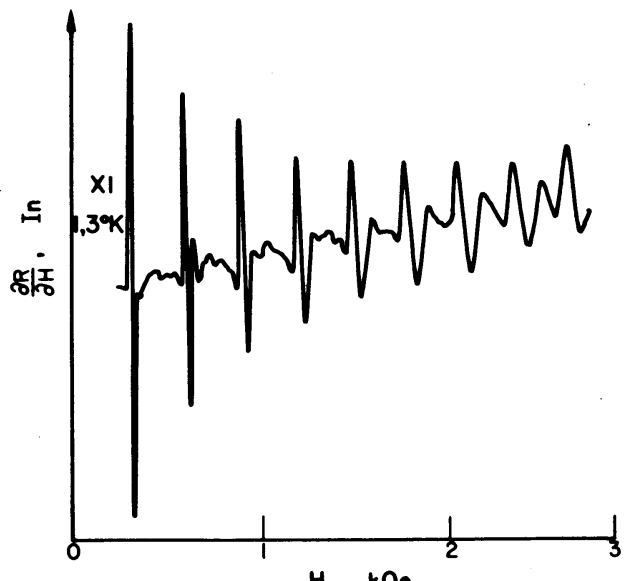

FIGURE 5

The dependence of the impedance derivative $d R / d H$ on magnetic field in indium (obtained by Gantmakher and Krylov [13]). The experimental conditions were given in the text.

probably connected with the fact that an approximate boundary condition has been used (the substitution of $\bar{v}_{z}$ for $v_{z}$ ). It is necessary to underline that in general the left side of the line is highly sensitive to the character of electron scattering at a metal surface. The skin-depth $\delta_{0}$ which was determined by the form and width of the curves appeared to be approximately equal to $3 \cdot 10^{-4} \mathrm{~cm}$. The experimental value $\delta_{0}$ measured at high frequency and recalculated to the frequency $3 \mathrm{Mc}$ gives $\delta_{0} \simeq 2.3 \cdot 10^{-4} \mathrm{~cm}$. The frequency dependence of the line width of the size effect $\Delta H \sim \delta_{0} \sim \omega^{-1 / 3}$ which has been studied $[13,16]$ agrees with the formula (28). Our calculations entirely confirm the conclusion that the line origin should be considered the left edge, not the maximum. Besides, we confirmed the fact $[14,16]$ that the extremum of one of the functions either $\partial R / \partial H$ or $\partial X / \partial H$ coincides with the region of rapid change of the other. However it is necessary to stress that such a connection between $\partial R / \partial H$ and $\partial X / \partial H$ (or $R$ and $X$ ) in the case of size effect has an approximate character, and is not an exact result of dispersion relations of Kramers-Kronig type (see also [4]).

The theoretical conclusion about the exponential increase of RSE line amplitude with change of inclination angle was already found in the first paper by Gantmakher and the author [12] together with an investigation of size effect from limiting points in Sn. There the method of measurement of the local value of the mean free path $l_{0}$ was proposed. With the help of this method the temperature dependence of $l_{0}$ has been measured in indium monocrystals [13]. The 
result which has been obtained in this paper

$$
1 / l(\mathrm{~T})=1 / l(0)+a T^{3}
$$

entirely confirms the conclusion of the anomalous skin-effect theory. According to $[19,20]$ the main role in the collision integral is played by the scattering out term for collisions of effective electrons with phonons. Therefore the collision frequency $v$ turns out to be proportional to the number of phonons, that is $T^{3}$.

Thus, all. the experimental data on the size effect is in excellent agreement with the theory. The line shape is essentially determined only by the character of the field distribution in a

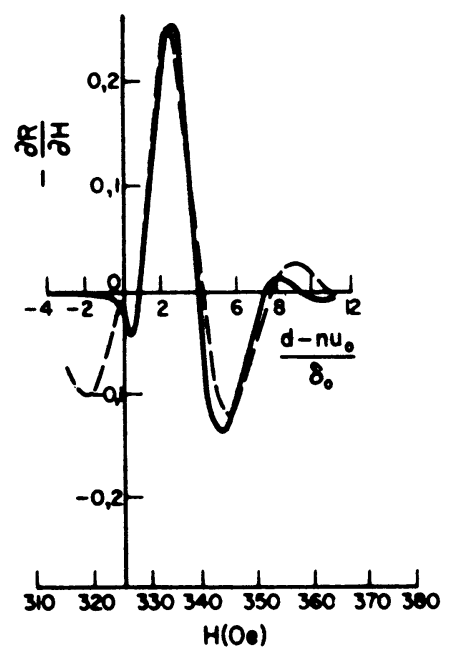

$H\left(O_{e}\right)$

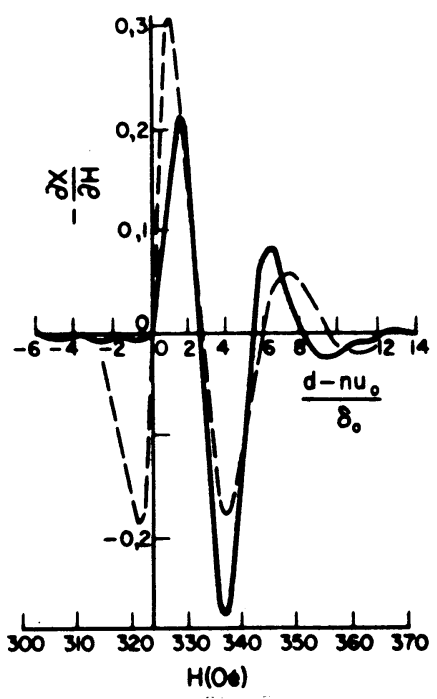

FIGURE 6

The comparsion of theoretical and experimental curves on the shape of the size effect line.

-

metal (only the function $T_{0}(z)$ is used in formula (30)). So the coincidence of the calculated and measured line shape is a direct confirmation of the anomalous skin-effect theory in metals.

\section{Acknowledgment}

The author is grateful to Gantmakher and Krulov for putting the experimental curves at his disposal. 


\section{References}

1. E. A. KANER, Dokl. Akad. Nauk SSSR 119, 471 (1958).

2. M.S., KHAIKIN, Zh, eksp. teor. Fiz. 41, 1773 (1961); 43, 59 (1962).

3. V.F. GANTMAKHER, Zh, eksp, teor, Fiz, 42, 1416 (1962); 44, 811 (1963).

4. E. A. KANER and V.L. FAL'KO, Zh. eksp. teor. Fiz. 51, 586 (1966).

5. V.F. GantmakheR, Prog. low Temp. Phys. (1966).

6. E. A. KANER and V. F. GANTMAKHER, Usp. fiz. Nauk. (1966).

7. E. A. KANER and V.G. SKOBOV, Usp. fiz. Nauk. 89, 367 (1966).

8. M. Ya. AZBEL', Zh. eksp, teor. Phys. 39, 400 (1960).

9. E. A. KANER, Zh. eksp. teor. Fiz. 44, 1036 (1963).

10. E. A. KANER and A.Ya. BlanC, J. Physics Chem. Solids 28, 1735 (1967).

11. V. F. GANTMAKHER, Zh. eksp. teor. Fiz. 43, 345 (1962).

12. V. F. GANTMAKHER and E.A. KANER, $Z h$. eksp. teor. Fiz. 45, 1430 (1963).

13. I.P. KRYLOV and V.F. GaNTMAKHeR, 10th Int. Congr. Low Temp. Phys. (Moscow, 1966); Zh. eksp. teor. Fiz, 51, 740 (1966).

14. I. P. KRYLOV, Zh. eksp. teor. Phys. Lett. 1, 24 (1965).

15. A. A. MARIACHIN and V.P. NABEREJNICH, Zh. eksp. teor. Fiz. 51 (1966).

16. J.F. KOCH and T.K. WAGNER, 10 th Int. Cong. Low Temp. Phys. (Moscow, 1966).

17. C. C. GRIMES, A.F. KIP, F. W. SPONG, R.A. STRADLING and P. PINCUS, Phys. Rev. Lett. 2, 455 (1963); P. W. SPONG and A. F. KIP, Phys. Rev. 137, A431 (1965).

18. E. A. KANER, Zh, eksp. teor. Phys. 34, 658 (1958).

19. M. Ya. AZBEL' and E. A. KANER, Zh, eksp. teor. Phys. 32, 893 (1957).

20. E. A. KANER and M. Ya. AZBEL', Zh. eksp. teor. Phys. 33, 1461 (1957). 